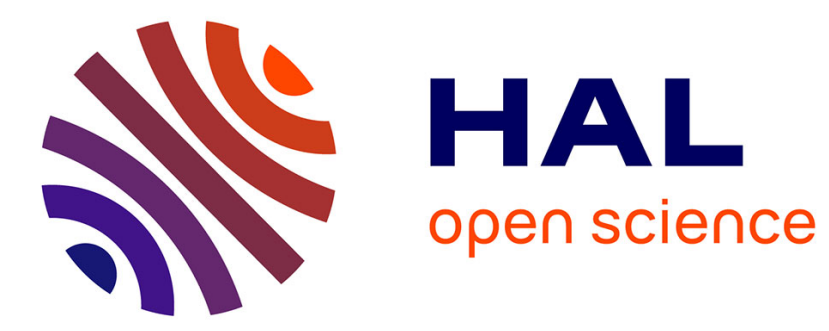

\title{
Temperature dependence of electrical resistivity in highly resistive alloys
}

F. Brouers

\section{To cite this version:}

F. Brouers. Temperature dependence of electrical resistivity in highly resistive alloys. Journal de Physique Lettres, 1978, 39 (18), pp.323-326. 10.1051/jphyslet:019780039018032300 . jpa-00231508

\section{HAL Id: jpa-00231508 https://hal.science/jpa-00231508}

Submitted on 1 Jan 1978

HAL is a multi-disciplinary open access archive for the deposit and dissemination of scientific research documents, whether they are published or not. The documents may come from teaching and research institutions in France or abroad, or from public or private research centers.
L'archive ouverte pluridisciplinaire HAL, est destinée au dépôt et à la diffusion de documents scientifiques de niveau recherche, publiés ou non, émanant des établissements d'enseignement et de recherche français ou étrangers, des laboratoires publics ou privés. 


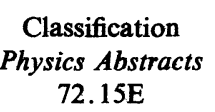

\title{
TEMPERATURE DEPENDENCE OF ELECTRICAL RESISTIVITY IN HIGHLY RESISTIVE ALLOYS
}

\author{
F. BROUERS \\ Institut für Theoretische Physik, Freie Universität, Berlin 33, Germany
}

(Reçu le 6 février 1978, révisé le 10 juillet 1978, accepté le 3 août 1978)

\begin{abstract}
Résumé. - Une discussion de travaux récents utilisant la théorie CPA et ATA de la conductibilité électrique des alliages fournit une expression de la résistivité conforme à une analyse générale de l'influence du couplage entre désordre thermique et de configuration dans les systèmes métalliques. Elle permet de mettre en évidence l'importance de l'élargissement de la bande dû aux phonons et aux fluctuations de spin sur la dépendance en $T$ de la résistivité des alliages cristallins, liquides et amorphes lorsque le niveau de Fermi se trouve dans un pic de densité d'état d ou f.
\end{abstract}

Abstract. - A discussion of recent works using the CPA and ATA theory of electrical conductivity of alloys at finite temperature yields an expression for the resistivity which is consistent with a general analysis of the influence of the coupling between thermal and configuration disorder in metallic systems.

It emphasizes the importance of band broadening induced by phonon or spin fluctuation disorder on the $T$-dependence of the resistivity and appears as an alternative model to describe the resistivity of crystalline, liquid or amorphous alloys when the Fermi level lies in a $\mathrm{d}$ or $\mathrm{f}$ density of states peak.

Recently Harris et al. [1] have discussed in the framework of the CPA theory [2-4] of conductivity the problem of the physical origin of the negative temperature of resistivity (TCR) observed in a number of highly resistive alloys.

Arguing that the CPA requires extensive selfconsistent calculations and therefore does not allow a detailed discussion of the mechanism responsible for a negative TCR, Harris et al. [1] derived an approximate expression for the resistivity in the average $T$-matrix approximation. The ATA is a non self-consistent approximation which can be regarded as a first iteration of the CPA equation [5]. In the dilute limit the self-energy has a simple analytical form. If one chooses for the electrons a semi-elliptic one-band (s-like) and an Einstein model for the phonon distribution, the resistivity due to substitutional and phonon scattering can be written in the form

$$
\rho=\rho_{0}+\rho_{\mathrm{ph}}+\rho_{\text {mix }}
$$

when both thermal and configuration averages are performed, Matthiessen's additivity rule is not fulfilled. A $T$-dependent mixing term appears as a consequence of the mixing of potential and phonon scattering. In Harris et al. [1] model and approximation, the $T$-independent residual resistivity reads :

$$
\rho_{0}=x \frac{\rho_{\mathrm{c}}}{g_{0}^{2}\left(E_{\mathrm{F}}\right)} f\left(V, E_{\mathrm{F}}\right)
$$

the phonon resistivity

$$
\rho_{\mathrm{ph}}=\frac{\rho_{\mathrm{c}}}{g_{0}^{2}\left(E_{\mathrm{F}}\right)} \alpha
$$

and a mixing term

$$
\rho_{\text {mix }}=-\rho_{0} \alpha N\left(V, E_{\mathrm{F}}\right) .
$$

In equation (2), $x$ is the impurity concentration, $\rho_{\mathrm{c}}$ a constant depending on the parameters of the lattice, $g_{0}\left(E_{\mathrm{F}}\right)$ is the density of states at Fermi level, $\alpha$ the mean square of the energy fluctuations due to the thermal displacement. This quantity is proportional to the Debye-Waller factor averaged over the $k$-vectors and to the square of the electron-phonon coupling constant. At $T$ equal or higher to the Debye temperature $\alpha$ is proportional to the absolute temperature $T$. The functions $f\left(V, E_{\mathrm{F}}\right)$ and $N\left(V, E_{\mathrm{F}}\right)$ vary with the scattering parameter $V$ (difference of local potential between host and impurity) and the position of the Fermi energy. $N\left(V, E_{\mathrm{F}}\right)$ strongly depends on these two quantities and tends asymptotically to unity for large values of $V$. Equations (1), (2a, $b$ and $c)$ are consistent with the result of a general analysis of Markowitz [6] who shows that the mixing between disorder and thermal scattering yields a mixing term proportional to the residual resistivity and to the Debye-Waller factor. However in the one-band dilute limit considered by Harris et al., $\rho_{0}$ is never high enough to induce a negative TCR. 
In this letter we show that applying Harris et al. arguments to the s-d model (hybridized s-d electrons plus d-electron-phonon coupling) which is more appropriate to describe transition metal conductivity and was used in reference [4] gives an expression for the resistivity in which the functions $N\left(V, E_{\mathrm{F}}\right)$ and $f\left(V, E_{\mathrm{F}}\right)$ have very simple physical interpretations : the mixing term results directly from the spreading of the d-density of states. It can be positive or negative according to the position of the Fermi level with respect to the peak of the d-impurity density of states.

We use the model of reference [4] and apply the same procedure as in Harris et al. [1]. We refer to Brouers and Vedyayev [3] for a general discussion of the CPA conductivity in this approach.

In the two-band model using a semi-elliptic band the resistivity of a tight binding band $A_{x} B_{1-x}$ alloy can be written [7]

$$
\rho=\rho_{\mathrm{c}} \frac{\Sigma_{\mathrm{s}}^{\prime \prime}\left(E_{\mathrm{F}}\right)}{g_{\mathrm{s}}^{3}\left(E_{\mathrm{F}}\right)\left(1+\frac{6 \Sigma_{\mathrm{s}}^{\prime \prime}\left(E_{\mathrm{F}}\right)}{\pi g_{\mathrm{s}}\left(E_{\mathrm{F}}\right)}\right)}
$$

where $\Sigma_{\mathrm{s}}^{\prime \prime}\left(E_{\mathrm{F}}\right)$ is the absolute value of the imaginary part of the s self-energy $\Sigma_{\mathrm{s}}$ induced by the d-disorder and $g_{0 \mathrm{~s}}\left(E_{\mathrm{F}}\right)$ is the $\mathrm{s}$ density of states. If one neglects the d-hopping and considers only a hybridization energy $\gamma$ between $\mathbf{s}$ and $\mathbf{d}$ electrons, one has (Levin and Ehrenreich [8]) :

$$
\Sigma_{\mathrm{s}}(z)=\frac{\gamma^{2}}{z-\Sigma_{\mathrm{d}}(z)} .
$$

In the ATA dilute limit $(x \ll 1)$, if the host energy level is taken as the energy origin

$$
\Sigma^{\prime \prime}(E) \simeq \frac{\gamma^{2}}{E} \operatorname{Im} \Sigma_{\mathrm{d}}(E)
$$

To calculate $\Sigma_{\mathrm{d}}$ resulting from an average over configuration and thermal disorder we follow Harris et al. arguments using the definition of the d-Green's function given in reference [3]. The thermal average gives rise to a Gaussian distribution of the d-energy levels of half-width $\alpha$. In the ATA dilute limit $\left.\Sigma_{\mathrm{d}} \simeq \ll t_{\mathrm{c}}\right\rangle_{\text {th }}$, the atomic $t$-matrix averaged over configuration and thermal disorder. In the thermal average one neglects terms of order $\alpha^{2}$ or higher. In the two-band model one has

$$
\rho=\rho_{0}^{\mathbf{A}}+\rho_{\mathrm{ph}}^{\mathbf{B}}+\rho_{\mathrm{ph}}^{\mathbf{A}} .
$$

When the Fermi level lies in the impurity d-band i.e. $E_{\mathrm{F}} \sim V$, the three contributions to the resistivity can be written in a very physically transparent form :

$$
\rho_{0}^{\mathrm{A}} \simeq \frac{x \rho_{\mathrm{c}}}{g_{0 \mathrm{~s}}^{3}\left(E_{\mathrm{F}}\right)} V_{\mathrm{sd}}^{2} g_{\mathrm{d}}^{\mathrm{A}}\left(E_{\mathrm{F}}\right) \simeq \frac{x \rho_{\mathrm{c}} \gamma^{2}}{g_{0 \mathrm{~s}}^{3}\left(E_{\mathrm{F}}\right)} \frac{\Gamma}{\left(E_{\mathrm{F}}-V\right)^{2}+\Gamma^{2}}
$$

is the impurity $T$-independent residual resistivity, the Lorentzian representing the d-impurity peak $g_{\mathrm{d}}^{\mathrm{A}}$. Its half-width is given by $\Gamma \simeq \pi \gamma^{2} g_{0 \mathrm{~s}}\left(E_{\mathrm{F}}\right), g_{0 \mathrm{~s}}\left(E_{\mathrm{F}}\right)$ is the density of states of the host. This result was already obtained by Brouers and Vedyayev [3]. Here

$$
V_{\mathrm{sd}}=\frac{V \gamma}{E_{\mathrm{F}}}
$$

The host phonon resistivity

$$
\rho_{\mathrm{ph}}^{\mathbf{B}} \simeq(1-x) \frac{\rho_{\mathrm{c}}}{g_{\mathrm{os}}^{2}\left(E_{\mathrm{F}}\right)} \cdot \pi \tilde{\alpha} \text { with } \quad \tilde{\alpha}=\frac{\alpha \gamma^{2}}{E_{\mathrm{F}}^{2}}
$$

is proportionnal to an effective electron-phonon interaction measuring the effect of d-electron-phonon coupling on the resistivity of s-electrons. The mixing term has a very simple form. It reads

$$
\rho_{\mathrm{ph}}^{\mathrm{A}}=-\rho_{0}^{\mathrm{A}}\left(g_{\mathrm{d}}^{\mathrm{A}}\right)^{2}\left(1-3\left(\frac{E_{\mathrm{F}}-V}{\Gamma}\right)^{2}\right) \alpha .
$$

This third contribution has the structure of the Markowitz mixing term. It is proportional to the residual resistivity and to the Debye-Waller factor. Its physical interpretation in this formalism is quite straightforward. If one starts from the residual resistivity term (7) and if one assumes that the effect of electron-phonon interaction is to give rise to a Gaussian broadening of the energy levels, the expression (7) for finite $T$ can be written as

$$
\begin{aligned}
& \rho_{0}^{\mathrm{A}}(T)=x \rho_{0} \gamma^{2} \frac{1}{\sqrt{2 \pi \alpha}} \times \\
& \quad \times \int_{-\infty}^{+\infty} \mathrm{e}^{-\eta^{2} / 2 \alpha}\left[\frac{\Gamma}{\left(E_{\mathrm{F}}-V+\eta\right)^{2}+\Gamma^{2}}\right] \mathrm{d} \eta
\end{aligned}
$$

expanding this expression in powers of $\alpha$ yields the following result

$$
\begin{aligned}
\rho_{0}^{\mathrm{A}}(T) \simeq x \rho_{\mathrm{c}} & \gamma^{2} \frac{\Gamma}{(E-V)^{2}+\Gamma^{2}} \times \\
\times & {\left[1-\alpha \frac{\Gamma^{2}\left[1-3\left(\frac{E-V}{\Gamma}\right)^{2}\right]}{\left[(E-V)^{2}+\Gamma^{2}\right]^{2}}\right] . }
\end{aligned}
$$

The $T$-dependent term proportional to $\alpha$ is exactly the contribution $\rho_{\mathrm{ph}}^{\mathrm{A}}$ (Eq. (9)).

This result shows that a possible negative contribution to the resistivity in the present s-d model arises as a direct consequence of the broadening of the impurity density of states with temperature.

At high temperature [1]

$$
\alpha \simeq \frac{\hbar^{2} k_{\mathrm{D}}^{2}}{M k_{3} \theta} \frac{T}{\theta}=\bar{w} \frac{T}{\theta}
$$

with $k_{\mathrm{D}}$ the Debye cut-off, $\theta$ the Debye temperature and $M$ the mass of the lattice ion. 
Defining

$$
\rho_{\mathrm{ph}}^{\mathbf{B}}=\rho_{1} \frac{T}{\theta}
$$

one can write the $T$-dependent part of the resistivity

$$
\rho(T)=\left(\rho_{1}-\rho_{0}\left(g_{\mathrm{d}}^{\mathrm{A}}\right)^{2}\left[1-3\left(\frac{E_{\mathrm{F}}-V}{\Gamma}\right)^{2}\right] \bar{w}\right) \frac{T}{\theta}
$$

the TCR is negative if

$$
\rho_{1}<\rho_{0}\left(g_{\mathrm{d}}^{\mathrm{A}}\right)^{2}\left[1-3\left(\frac{E_{\mathrm{F}}-V}{\Gamma}\right)^{2}\right] \bar{w} .
$$

The condition is fulfilled if $\rho_{0}$ and $g_{\mathrm{d}}^{\mathrm{A}}\left(E_{\mathrm{F}}\right)$ are high enough and if the Fermi level lies in the centre of the d-peak i.e. if

$$
\left|E_{\mathrm{F}}-V\right|<\Gamma / \sqrt{3}
$$

In all these calculations we have neglected the effect of a possible hybridization gap for two reasons, we assume that due to crystal symmetry and other scattering mechanisms this gap is unphysical and we suppose that the Fermi level does not lie in a region where unphysical singularities can occur in the selfenergy.

If we compare these results and the one in the oneband model some remarks are in order. $\rho_{0}$ is still proportional to the concentration of impurities, $g_{\mathrm{d}}^{\mathrm{A}}\left(E_{\mathrm{F}}\right)$ can be much higher than the $N\left(V, E_{\mathrm{F}}\right)$ function and the condition (16) places higher constraints on the position of the Fermi level. However it has been shown that in the s-d model the ATA is an excellent approximation and that the dilute limit can be extrapolated to higher concentrations [9].

The results of this letter should be considered in a more general perspective. A Gaussian broadening of energy levels can arise as the consequence of other mechanisms. For instance in strongly paramagnetic systems, Hertz and Klenin [10] have shown that due to spin fluctuations the density of states is averaged over a width $\sqrt{U k T}$ :

$$
\langle g(E)\rangle=\int_{-\infty}^{+\infty} \frac{\alpha \eta}{\sqrt{2 \pi \xi^{2}}} g(E+\eta) \mathrm{e}^{-\eta^{2} / 2 \xi^{2}}
$$

with $\xi \simeq \sqrt{U k T}$ and the present formalism can be used to analyse the influence of this smearing on the $T$-dependence of the resistivity. It is formally identical to the phonon problem if one replaces $\alpha$ by $\xi^{2}$.

Other kind of interactions can give rise to similar effects for instance if a topological disorder is simulated by a Lorentzian distribution of energy levels (Lloyd model) [11] of half-width $\Delta$, the residual resistivity due to configuration and topological disor- der will be proportional to a Lorentzian of width $(\Gamma+\Delta)$. If $\Delta$ is $T$-dependent the mixing term can increase or decrease with $T$ according to the position of the Fermi level with respect to the d-density of states peak. To first order in $\Delta$ one has

$$
\rho \propto \frac{\Gamma}{E^{2}+\Gamma^{2}}\left(1+\frac{\Delta}{\Gamma} \frac{E^{2}-\Gamma^{2}}{E^{2}+\Gamma^{2}}+\mathrm{O}\left(\Delta^{2}\right)\right) .
$$

If $|E|<\Gamma$ one obtains a decrease of the resistivity with $T$.

Conclusions. - The calculations presented in this letter show that in alloys the deformation of the density of states with $T$ induced by electron-phonon interaction or spin-fluctuations, being more important than the Fermi-smearing considered previously [12], can give rise to a negative $T$-coefficient of the resistivity as this has been observed in highly resistive alloys. In alloy liquids the standard theory is based on the Ziman's theory [13] of simple liquid metals. It has been extended to metals with d-resonant scattering by Evans et al. [14, 15]. In that case

$$
\rho \propto\left(k_{\mathrm{F}}^{2} E_{\mathrm{F}}\right)^{-1} a\left(2 k_{\mathrm{F}}\right) \frac{\Gamma}{\left(E-E_{\mathrm{res}}\right)^{2}+\Gamma^{2}}
$$

where $\mathrm{F}$ refers to the Fermi level and $E_{\text {res }}$ is the resonant energy. When $2 k_{\mathrm{F}} \sim k_{\mathrm{p}}$ the wave-vector corresponding to the first peak of the structure factor $a(k)$, the broadening of $a(k)$ with $T$ can yield a negative TCR in liquids. However there is also a strong correlation between the sign of the TCR and the magnitude of resistivity in crystalline and amorphous alloys [16] and our model accounts also for this observation. If in concentrated alloys the Fermi level lies in a peak of the d-density of states it is plausible that in the liquid state since the system exhibits both topological and configurational disorder the broadening of $a(k)$ and of the d-states could be of equal importance.

The model discussed here can hardly be used when the mean free path becomes comparable to the interatomic spacing. In that case as this is discussed by Allen et al. [17] and Fisk and Webb [18] saturation effects occur and the modification of the band structure by impurities and thermal disorder as discussed in this letter is not probably the essential factor governing the $T$-dependence of the resistivity.

The considerations we have developed can be extended to compounds with rare-earth constituents for example to mixed valence systems where anomalous behaviour of the $T$-dependence of the resistivity has been observed. According to the position of the Fermi level in the f narrow band, the resistivity can increase or decrease with $T$. The valence can change with $T$ and this can give rise to a variety of possible behaviours as this is observed [19]. 


\section{References}

[1] Harris, R., Shalmon, M., Zuckermann, M., Phys. Rev. (1978) to be published.

[2] Chen, A., Weisz, G., Sher, A., Phys. Rev. B 5 (1972) 2897.

[3] Brougrs, F., Vedyayev, A. V., Phys. Rev. B 5 (1972) 348.

[4] Brouers, F. and Brauwers, M., J. Physique Lett. 36 (1975) L-17.

[5] Schwartz, L., Brouers, F., Vedyayev, A. V. and EhrenREICH, H., Phys. Rev. B 4 (1971) 3383.

[6] Markowitz, D., Phys. Rev. B 15 (1977) 3617.

[7] Bhattacharya, A. K., Brauwers, M., Brouers, F. and Joshi, S. K., Phys. Rev. B 13 (1976) 5214.

[8] Levin, K. and Ehrenreich, H., Phys. Rev. B 3 (1971) 4172.

[9] Brouers, F. and Vedyayev, A. V., J. Phys. F3(1973) 127.

[10] Hertz, J. A. and Klenin, M. A., Phys. Rev. B 10 (1974) 1084.

[11] LLOYd, P., J. Phys. C (1969) 1717.

[12] Cohen, R. W., Cody, G. D. and Halloran, J. J., Phys. Rev. Lett. 19 (1967) 840.

[13] Ziman, J. M., Philos. Mag. 6 (1961) 1013 ;

Ziman, J. M., Adv. Phys. 16 (1967) 551.

[14] Evans, R., Gyorffy, B. L., Szabo, N. and Ziman, J. M., Proc. Int. Conf. Liquid Metals (1972), p. 319.

[15] Busch, G., GüNTHERODT, H. J., Solid State Phys. 29 (1974) 235.

[16] Moou, J. H., Phys. Status Solidi (a) 17 (1973) 521.

[17] Allen, P. B., Phys. Rev. Lett. 37 (1976) 1638 ;

Wiesmann, H., Gurvitch, M., Lutz, H., Ghosh, A., Schwarz, B., Strogin, M., Allen, P. B. and HalLEY, J. W., Phys. Rev. Lett. 38 (1977) 782.

[18] Fisk, Z. and WebB, G. W., Phys. Rev. Lett. 36 (1976) 1084.

[19] Valence Instabilities and Related Narrow Bands Phenomena. Ed. R. D. Parks (Plenum Press) 1977. 